\title{
Association Between NOX4 And Nrf2 Genes in Non-Small-Cell Lung Carcinoma: A Case-Control Study
}

\author{
Nasim Bakhtiyari ${ }^{1}$, Akbar Sharifi ${ }^{1}$, Younes Aftabi ${ }^{1}$, Neda Gilani ${ }^{2}$, Venus Zafari ${ }^{1}$, \\ Atefeh Ansarin ${ }^{1}$, Ensiyeh Seyedrezazadeh*1
}

\begin{abstract}
Background: Epithelial malignancy in lung cancer, which is initiated with myofibroblast differentiation and remodeling, promotes hypoxia and intracellular ROS generation most affected by the prototypical enzyme, NADPH oxidase 4 (NOX4). In addition, nuclear factor erythroid 2-related factor 2 (Nrf2) acts as a critical transcription factor by stimulating antioxidant proteins as redox homeostasis regulators. The aim of this study was to investigate a possible correlation between lung tissue NOX4 and Nrf2 genes (NOX4 and Nrf2) mRNA expression and bronchoalveolar lavage fluid (BALF) protein expression in non-small-cell lung carcinoma (NSCLC) patients.

Methods: Samples from 25 patients with various NSCLC types and stages and 20 healthy controls were collected. NOX4 and Nrf2 mRNA were measured by qRT-PCR, and protein by western blot analysis.

Results: NOX4 mRNA and protein expression was significantly up-regulated in NSCLC patients' lung tissues and BALFs ( $\mathrm{p}=0.03$ and 0.01 , respectively). In addition, by adjusting for age, sex, and NSCLC types and stages, a significant and positive correlation was observed between NOX4 and Nrf2 mRNA expression $(\mathrm{r}=$ $0.927, \mathrm{p}=0.001)$. This was also true when not adjusted as above $(\mathrm{r}=0.944, \mathrm{p}<0.001)$.

Conclusions: NOX4 mRNA and protein expression is significantly up-regulated in NSCLC patients' lung tissues and BALFs, and NOX4 and Nrf2 mRNA expression is positively correlated in NSCLC tissues.
\end{abstract}

Keywords: Gene expression, Non-small-cell lung cancer, NOX4, Nrf2.

\section{Introduction}

Lung cancer, a major health problem affecting millions of people worldwide, is the leading cause of cancer-related mortality in humans (1). It is a complex and heterogeneous malignancy which, based on tumor histology, is classified into two main types; (I) non-small-cell, and (II) small cell, lung cancer (NSCLC and SCLC, respectively). NSCLC is the most common type with approximately $85 \%$ of all cases that contribute to metastatic lung cancers (2). Common NSCLC subtypes include squamous cell carcinoma, large cell carcinoma, and adenocarcinoma. Identifying the biological pathways and genetic abnormalities involved in lung cancer is essential for effective treatment (3). Hypoxia is a critical situation that influences the activity of important carcinogenic pathways. Hypoxia, tumor invasion, and metastasis contribute to the generation of TGF- $\beta 1$, induced by the epithelial-mesenchymal transition (EMT) that is developed by losing cell-to-cell contact and transforming to mesenchymal properties. TGF- $\beta 1$ is a multifunctional cytokine that induces its biological effects through both canonical and non-canonical pathways $(4,5)$. Growing evidence suggests that the Smadindependent, non-canonical pathway, mediates p38 mitogen-activated protein kinase (P38 
MAPK) and the phosphatidylinositol 3kinase/Akt/mammalian target of rapamycin (PI3/AKT/mTOR) signaling pathway, and is thought to promote cancer progression through EMT involved in migration, invasion, and metastasis (5-7). İn this process, the hypoxiainduced EMT is involved so that TGF- $\beta$ produces reactive oxygen species (ROS) mediated by NOX4 upregulation (5). ROS play an important role in the early stage of hypoxiainduced EMT in various cancers, including lung cancer $(8,9)$. ROS contribute to cancer development by modulating transcription and signal transduction pathways, and increasing replication errors and genomic instabilities (10). NADPH oxidase (NOX) complexes are major sources of endogenous non-mitochondrial ROS production in normal and cancerous cells. NOX 4, a member of the NOX family, is highly expressed and constitutively activated in airway and alveolar epithelial cells (11). NOX4 is activated in response to pulmonary disorders including ischemia and hypoxia (12) and, in relation to TGF- $\beta$, produces ROS that are regulated via various transcription factors (TFs) including E2F, Nrf2, hypoxia-inducible factor 1alpha (HIF-1a), NF-кB, and STAT3 through a mechanism that involves binding to the NOX4 DNA promoter site $(8,13)$. One of these TFs is a basic leucine zipper called nuclear factor erythroid 2-related factor 2 ( Nrf2), which, under special conditions, such as hyperoxia and oxidative stress, binds to antioxidant response elements on the NOX4 promoter and regulates its expression (14).

Recent evidence suggested that Nrf2 can upregulate SOD and GST, which can deplete ROS and lipid peroxidation (15). On the other hand, PI3K/AKT mediated Nrf2 up-regulation produces redox conditions, promoting cancer cell migration and survival (16). For example, using various NSCLC cell lines, Wu et al. reported that NOX4-derived $\mathrm{H}_{2} \mathrm{O}_{2}$ upregulates Nrf2 activity and expression (17). Here, we aimed to analyze lung tissue for Nrf2 and NOX4 mRNA and BALF for Nrf2 and NOX4 proteins and their possible associations in patients who underwent lung bronchoscopies.

\section{Materials and Methods \\ Study subjects, tissue, and lavage sampling}

Patients with suspected cases of lung carcinoma who attended the five lung and respiratory disease offices in Tabriz, Iran, between Sept 2016 and Feb 2018 were subjected to diagnostic tests.

Patients with tuberculosis or hemoptysis, those with prior radiotherapy or chemotherapy, and those unwilling to participate were excluded from the study. Patient medical histories were reviewed and interpreted independently by both a pulmonary pathologist and a lung disease specialist. Those with NSCLC who agreed to participate were included in the study and their disease stages were determined.

Forty-five subjects underwent bronchoscopic needle biopsy and histopathological diagnosis at Imam Reza and Shahid Madani hospitals following previously described methods (18). The collected tissue samples were suspended immediately in RNAlater (Qiagen, Cat. No. 76106), and the BALF specimens were collected in $50 \mathrm{ml}$ sterilized tubes and stored at $-80{ }^{\circ} \mathrm{C}$. The study protocol was approved by the Ethics Committee of the Tabriz University of Medical Sciences (IR.TBZMED.REC.1396.371) and all specimens were obtained from contributors with informed and signed consent.

\section{RNA extraction and real-time PCR}

Total RNA was extracted from tissue samples using the RNeasy Mini Kit (Qiagen, Cat. No. 74104) according to the manufacturer's instructions, and its quantity and quality were assessed on a Thermo Scientific ${ }^{\mathrm{TM}}$ NanoDrop ${ }^{\mathrm{TM}}$ One Spectrophotometer and by $1.5 \%$ agarose gel electrophoresis, respectively. Also, for cDNA synthesis a Thermo Scientific ${ }^{\text {TM }}$ K1691 kit was used following the manufacturer's instruction. The specific primers (Table 1) for target genes NOX4 and Nrf2 were designed using Primer3, Gene Runner version 6.5.52, and NCBIBLAST programs, and for internal control, 
the GAPDH gene primer sequences were obtained from a previous report (19-21). The real-time PCRs were performed at least in triplicate using a 48 well Step One ${ }^{\mathrm{TM}}$ RealTime PCR System and RealQ Plus Master Mix Green kit (Ampliqon A/S, Denmark) with the following conditions: $94{ }^{\circ} \mathrm{C}$ for 15 min, 40 amplification cycles consisting of 94 ${ }^{\circ} \mathrm{C}$ for $15 \mathrm{sec}$, annealing temperature (Table
1) for $30 \mathrm{sec}$, and $72{ }^{\circ} \mathrm{C}$ for $35 \mathrm{sec}$. Melting curves were then determined with temperatures ranging from 60 to $95^{\circ} \mathrm{C}$. SYBR Green reagents were used for all real-time PCRs. Genes expression was analyzed based on the cycle threshold $(\mathrm{Ct})$ and relative expression levels were determined as $2^{-}$ $\left[{ }^{\Delta \Delta} \mathrm{C}(\mathrm{t})\right]$. Each gene's expression was normalized to $G A P D H$.

Table 1. Primer sequences and Real-Time PCR conditions.

\begin{tabular}{|c|c|c|c|}
\hline Gene & Primers & Band & Annealing temperature $\left({ }^{\circ} \mathrm{C}\right)$ \\
\hline NOX4 & $\begin{array}{l}\text { F: TTTAGATACCCACCCTCCCG } \\
\text { R: AGCTTGGAATCTGGGCTCTT }\end{array}$ & $181 \mathrm{bp}$ & 64 \\
\hline$N r f 2$ & $\begin{array}{l}\text { F: TCTCCACAGAAGACCCCAAC } \\
\text { R: TGCTTTCAGGGTGGTTTTGG }\end{array}$ & $234 \mathrm{bp}$ & 64 \\
\hline GAPDH & $\begin{array}{l}\text { F: GACCCCTTCATTGACCTCAACTAC } \\
\text { R: TCGCTCCTGGAAGATGGTGATGG }\end{array}$ & $138 \mathrm{bp}$ & 62 \\
\hline
\end{tabular}

\section{Western-blot analysis}

For immunoblotting, the BALF samples were lysed using the lysing buffer and the lysates were centrifuged at $13300 \mathrm{~g}$. The total protein concentration in the supernatants was measured by Bradford assay. The proteins were separated by SDS-PAGE and transferred to PVDF membranes. The membranes were blocked with $2 \%$ low-fat milk in Trisbuffered saline and then incubated for 16-18 $\mathrm{h}$ with commercially available $\beta$-Actin (C4: sc-47778), NOX4 (3H2G11: sc-517188), and $N r f 2$ (A-10: $\quad$ sc-365949) primary antibodies. The membrane was washed in washing buffer, incubated with the appropriate HRP-conjugated secondary antibody (m-IgGк BP-HRP: sc-516102), and bands were visualized by enhanced chemiluminescence. Densitometry was performed using NIH Image/Image J software. All antibodies were purchased from Santa Cruz Biotechnology Inc.

\section{Statistics analysis}

Kolmogorov-Smirnov's with kurtosis and skewness indices tests were applied to assess normality. Unpaired t-test was performed to compare expression of the target genes between the case and control groups. Results were expressed as means \pm SDs, and a twotailed $\mathrm{p}<0.05$ indicated statistical significance. Additionally, the Pearson product-moment correlation test was used to test the correlation between the target genes' expression. Also, a partial correlation test was used to analyze the association of NOX4 and Nrf2 expression controlling for age, sex, and NSCLC types and stages. All analyses were performed using SPSS software, version 16.

\section{Results}

The participants' general characteristics are shown in Table 2a. Of the 45 participants, 25 were diagnosed as NSCLC cases including 8 adenocarcinoma, 10 squamous, and 7 largecell cancerous patients, while 20 were diagnosed as noncancerous, and used as the control group (Table 2a). The frequency of each stage in the NSCLC-diagnosed patients is shown in Table $2 b$.

The quality and quantity of extracted RNA were confirmed by the A260/280 (1.8-2) and 230/260 (1.6-1.8) ratios, and the identification of sharp bands of the ribosomal RNAs on an agarose gel. NOX4 expression was significantly greater in NSCLC patient tissue samples than in controls $(\mathrm{p}=0.03)$ (Fig. 1A). Although the Nrf2 expression was greater in NSCLC patients than in controls, this difference was not significant $(p=0.163)$ (Fig 1a). 
Table 2. General characteristic of study subjects (a) and frequency of cancer types and stages (b).

\begin{tabular}{|c|c|c|c|c|}
\hline Characteristic & Case & & Control & \\
\hline Age $($ Mean \pm SD) & $58.76(16.43)$ & & $58.05(14.71)$ & \\
\hline$\overline{\mathrm{Sex}}$ & number $(\%)$ & & & \\
\hline Male & $18(72 \%)$ & & & \\
\hline \multicolumn{5}{|l|}{ Cancer type } \\
\hline Adenocarcinoma & $8(32 \%)$ & & & \\
\hline Squamous & $10(40 \%)$ & & & \\
\hline Large Cell & $7(28 \%)$ & & & \\
\hline \multicolumn{5}{|l|}{ Cancer stage $n(\%)$} \\
\hline $\mathrm{I}$ & $1(4 \%)$ & & & \\
\hline$\underline{\mathrm{II}}$ & $9(36 \%)$ & & & \\
\hline III & $8(32 \%)$ & & & \\
\hline \multirow[t]{2}{*}{ IV } & $7(28 \%)$ & & & \\
\hline & & b & & \\
\hline \multirow[t]{2}{*}{ Cancer type } & Stages & & & \\
\hline & I & II & III & IV \\
\hline Adenocarcinoma & 0 & 3 & 4 & 1 \\
\hline Squamous cells & 0 & 4 & 3 & 3 \\
\hline Large cells & 1 & 2 & 1 & 3 \\
\hline
\end{tabular}

a

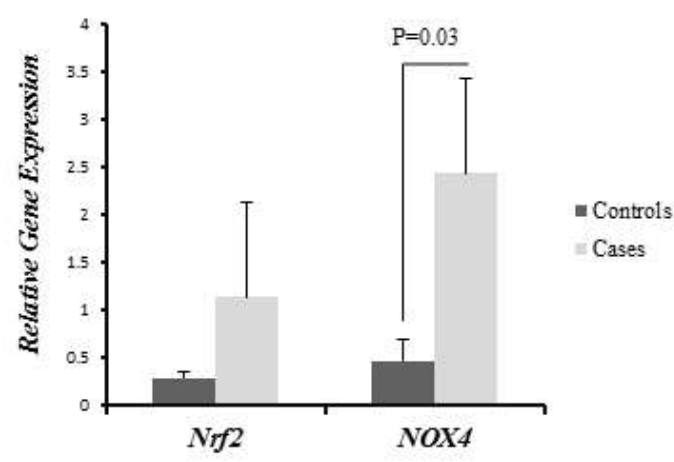

C

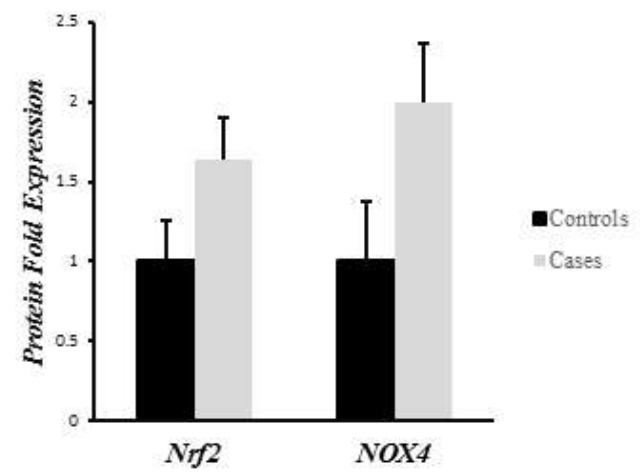

b

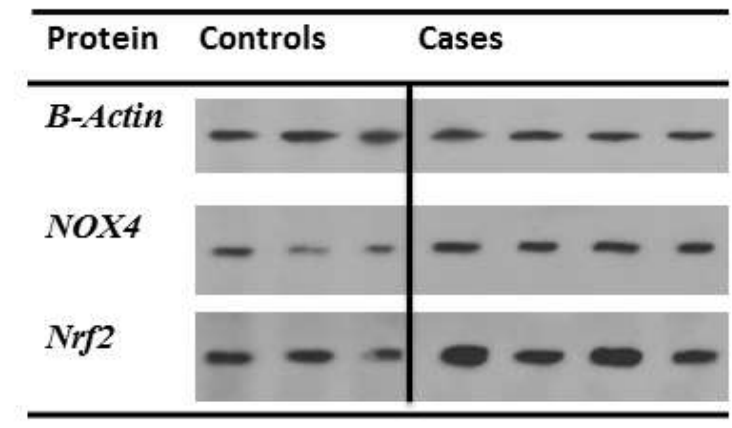

Fig. 1. Nrf2 and NOX4 expression in tissue samples and abundance of their associated proteins in the BALFs by RT-PCR and western blotting. (a) Nrf2 and NOX4 expression in the NSCLC patient tissue samples was greater than in controls, however, the increase was statistically significant only for $N O X 4$ ( $\mathrm{p}=0.03$ ). (b) The protein levels, by western blotting. (c) Densitometry revealed greater abundances of both Nrf2 and NOX4 protein in patient BALFs than in controls, however as with the mRNAs, the difference was significant only for NOX4 $(\mathrm{p}=0.01)$. 
Western blot and densitometry showed that the NOX4 protein level was significantly greater in the in the NSCLC patient BALF specimens than in controls $(\mathrm{p}=0.01)$ (Figs. $1 \mathrm{~b}$ and 1c). Although the $N r f 2$ protein content was greater in NSCLC patients than in controls, the difference was not statistically significant.

The Pearson product-moment correlation test without adjusting for covariates showed a significant positive correlation between $N r f 2$ and NOX4 expression ( $\mathrm{r}=0.944$, $\mathrm{p}<0.001$ ) in NSCLC tissue samples. A partial correlation test adjusted for age, sex, and NSCLC types and stages also showed a significant positive correlation between $N r f 2$ and NOX4 expression ( $\mathrm{r}=0.927$, $\mathrm{p}=0.001$ ), and also when not adjusted for those factors $(r=0.944, p<0.001)$.

\section{Discussion}

In this study, we found significantly greater NOX4 expression and non-significantly greater $N r f 2$ expression in NSCLC patient lung tissue than in that of controls. Moreover, similar results were observed for their respective proteins in the BALFs. In addition, we found a significant correlation between Nrf2 and NOX4 expression in the NSCLC patient lung tissues.

In response to hypoxia and inflammatory conditions in various cancer cells, including NSCLC, reactive oxygen and nitrogen species (RONS) are induced by the main ROS producer, NOX4, and nitric oxide synthases (22). RONS have detrimental effects on proteins, DNA, and organelles. Indeed, NOX4induced oxidative stress promotes hypoxiainduced EMT through the driving force of TGF $\beta 1$ (22). Based on the physiological condition, concomitant with the accumulation of RONS, the enzymatic and non-enzymatic endogenous antioxidant systems are initiated to maintain cellular redox homeostasis. Recent evidence suggests that the redox-sensitive residues in regulatory proteins, including cysteine oxidation/S-sulfenylation/Sglutathionylation/S-nitrosylation and tyrosine nitration, activate intracellular signaling pathways and transcription factors that promote cell proliferation, migration, and survival (22). These oxidative/nitrosative modifications activate some intracellular transcription effectors, including $N r f 2$ as a master of the antioxidant response regulator in PI3K/Aktmediated signaling pathways (22). Recent studies indicate that $N r f 2$ exhibits a dual role in cancer progression (23). During the early stage of cancer, under oxidative stress, Nrf2 dissociates from Keap1, translocates into the nucleus, and binds to the antioxidant response element (ARE), which promotes downstream antioxidant protein expression. Nrf2 overexpression also substantially reduces tumor cell sensitivity to chemotherapeutic agents (22).

Silva et al. reported that unbalanced levels of $N r f 2 /$ Keap1, in which $N r f 2$ is increased, act as a biomarker for cisplatin resistance in LC cell lines (24). An in vivo study demonstrated that oncogenic KRAS activates the NOX complex, and subsequent downstream Nrf2-dependent antioxidant mechanisms, supporting tumor progression (25). Wu et al. found that Nrf2 confers apoptosis resistance in NOX4-overexpressed NSCLC cells, and the enhancement effect of NOX4 on cell growth is reversible by Nrf2 inhibition (17).

As discussed above, oxidative/nitrosative modifications are a major factor in cancer initiation and progression. İn this line, NOX4 and Nrf2 play key roles in oxidative stress regulation and the EMT process via various signaling pathways. Based on our findings, increased NOX4 and Nrf2 mRNA and protein, and also their significant positive correlation, indicated that they are significant factors in lung cancer proliferation and progression.

Some limitations should be addressed regarding the present study. Because of the difficulty of sampling via biopsy from lung and BALF, we had limited tissue and BALF specimens. for that reason, we were unable to measure some of the agents implicated in NOX4 and Nrf2 function, for exampleoxidative stress and TGF- $\beta 1$. 
Moreover, our subject number was relatively small.

In conclusion, we found upregulated $N O X 4$ and $N r f 2 \mathrm{mRNA}$ and protein in lung tissue and BALF of NSCLC patients relative to controls. İn addition, a strongly positive association was observed between NOX4 and Nrf2. Considering the implication of NOX4 and Nrf2 in key carcinogenic signaling pathways, our findings suggest that these biomarkers may be candidates as NSCLC diagnostic and treatment targets. Moreover, studying the molecular aspects of gene-gene interactions regarding $N r f 2$ and $N O X 4$ could provide

\section{References}

1. Vergnenegre A, Chouaid C. Review of economic analyses of treatment for non-small-cell lung cancer (NSCLC). Expert Rev Pharmacoecon Outcomes Res 2018;18(5):519-528.

2. Siegel RL, Miller KD, Jemal A. Cancer statistics, 2018. CA Cancer J Clin 2018;68(1):7-30.

3. Esfandi F, Fallah H, Arsang-Jang S, Taheri M, Ghafouri-Fard S. The Expression of CCAT2, UCA1, PANDA and GHET1 Long Non-coding RNAs in Lung Cancer. Rep Biochem Mol Biol 2019;8(1):36-41.

4. Li H, Peng C, Zhu C, Nie S, Qian X, Shi Z, et al. Hypoxia promotes the metastasis of pancreatic cancer through regulating NOX4/KDM5Amediated histone methylation modification changes in a HIF1A-independent manner. Clin Epigenetics 2021;13(1).

5. Kim SJ, Kim YS, Kim JH, Jang HY, Ly DD, Das R, et al. Activation of ERK1/2-mTORC1NOX4 mediates TGF- $\beta 1$-induced epithelialmesenchymal transition and fibrosis in retinal pigment epithelial cells. Biochemical and Biophysical Research Communications. 2020;529(3):747-752.

6. Witte D, Bartscht T, Kaufmann R, Pries R, Settmacher U, Lehnert H, et al. TGF- $\beta 1$-induced cell migration in pancreatic carcinoma cells is RAC1 and NOX4-dependent and requires RAC1 and NOX4-dependent activation of p38 MAPK. Oncol Rep. 2017;38(6):3693-3701.

7. El-Sayed Ibrahim N, Morsy H, Abdelgwad M. The Comparative Effect of Nisin and Thioridazine valuable information on the molecular regulation of the oxidative pathways in lung cancer pathobiology.

\section{Acknowledgements}

The help of staffs in the Bronchoscopy Room in Imam Reza Hospital, Tabriz, Iran is greatly appreciated. This study was funded by the Tuberculosis and Lung Diseases Research Center, Tabriz University of Medical Sciences, Tabriz, Iran (Grant No: 57908).

All authors declare they have no conflicts of interest.

as Potential Anticancer Agents on Hepatocellular Carcinoma. Rep Biochem Mol Biol. 2021;9(4):452-462.

8. Meitzler JL, Konaté MM, Doroshow JH. Hydrogen peroxide-producing NADPH oxidases and the promotion of migratory phenotypes in cancer.

Arch Biochem Biophys. 2019; 675:108076

9. Filaire E, Dupuis C, Galvaing G, Aubreton S, Laurent H, Richard R, et al. Lung cancer: what are the links with oxidative stress, physical activity and nutrition. Lung Cancer. 2013;82(3):383-9.

10. Reuter S, Gupta SC, Chaturvedi MM, Aggarwal BB. Oxidative stress, inflammation, and cancer: how are they linked? Free Radic Biol Med. 2010;49(11):1603-1616.

11. Hollins F, Sutcliffe A, Gomez E, Berair R, Russell R, Szyndralewiez C, et al. Airway smooth muscle NOX4 is upregulated and modulates ROS generation in COPD. Respir Res. 2016;17(1):84.

12. Parent RA. Comparative Biology of the Normal Lung (Second Edition). San Diego: Academic Press; 2015.

13. Guo S, Chen X. The human Nox4: gene, structure, physiological function and pathological significance. J Drug Target. 2015;23(10):888-96.

14. Bernard K, Hecker L, Luckhardt TR, Cheng G, Thannickal VJ. NADPH oxidases in lung health and disease. Antioxid Redox Signal. 2014;20(17):2838-53.

15. Jahan N, Chowdhury A, Li T, Xu K, Wei F, Wang S. Neferine improves oxidative stress and 
apoptosis in benign prostate hyperplasia via Nrf2ARE pathway. Redox Rep. 2021;26(1):1-9.

16. Koundouros N, Poulogiannis G. Phosphoinositide 3-Kinase/Akt Signaling and Redox Metabolism in Cancer. Front Oncol. 2018;8:160.

17. Wu Q, Yao B, Li N, Ma L, Deng Y, Yang Y, et al. Nrf2 mediates redox adaptation in NOX4overexpressed non-small cell lung cancer cells. Exp Cell Res. 2017;352(2):245-254.

18.Cappellari JO, Haponik EF. Bronchoscopic nee dle aspiration biopsy. Am J Clin Pathol. 2000;113(5 Suppl 1): S97-108.

19. Untergasser A, Cutcutache I, Koressaar T, Ye J, Faircloth BC, Remm M, et al. Primer3--new capabilities and interfaces. Nucleic Acids Res. 2012;40(15):e115.

20. Johnson M, Zaretskaya

I, Raytselis Y, Merezhuk Y, McGinnis S, Madden TL. NCBI BLAST: a better web interface. Nucleic Acids Res. 2008;36(Web Server issue):W5-9.

21.Aftabi Y, Zarredar H, Sheikhi M, Khoshkam Z,
A HC. Induction of AHR Gene Expression in Colorectal Cancer Cell Lines by Cucurbitacin D, E, and I. J Cell Mol Res 2019;10:67-75.

22. Moldogazieva NT,

Lutsenko

SV, Terentiev AA. Reactive Oxygen and Nitrogen Species-Induced Protein Modifications: Implication in Carcinogenesis and Anticancer Therapy. Cancer Res 2018;78(21):6040-6047.

23. Li J, Xiong C, Xu P, Luo Q, Zhang R. Puerarin induces apoptosis in prostate cancer cells via inactivation of the Keap1/Nrf2/ARE signaling pathway. Bioengineered 2021;12(1):402413.

24. Silva MM, Rocha CRR, Kinker GS, Pelegrini AL, Menck CFM. The balance between NRF2/GSH antioxidant mediated pathway and DNA repair modulates cisplatin resistance in lung cancer cells. Sci Rep 2019;9(1):17639.

25. Lim JKM, Leprivier G. The impact of oncogenic RAS on redox balance and implications for cancer development. Cell Death Dis 2019;10(12):955. 